\title{
Coulomb Energy Density Functionals for Nuclear Systems: Recent Studies of Coulomb Exchange and Correlation Functionals
}

\author{
Tomoya Naito ${ }^{1,2, *}$, Ryosuke Akashi ${ }^{1}$, Gianluca Colò ${ }^{3,4}$, Haozhao Liang ${ }^{2,1}$, and Xavier Roca-Maza ${ }^{3,4}$ \\ ${ }^{1}$ Department of Physics, Graduate School of Science, The University of Tokyo, Tokyo 113-0033, Japan \\ ${ }^{2}$ RIKEN Nishina Center, Wako 351-0198, Japan \\ ${ }^{3}$ Dipartimento di Fisica, Università degli Studi di Milano, Via Celoria 16, 20133 Milano, Italy \\ ${ }^{4}$ INFN, Sezione di Milano, Via Celoria 16, 20133 Milano, Italy
}

\begin{abstract}
The Coulomb exchange and correlation energy density functionals for electron systems are applied to nuclear systems. It is found that the exchange functionals in the generalized gradient approximation provide agreements with the exact-Fock energy with one adjustable parameter within a few dozen keV accuracy, whereas the correlation functionals are not directly applicable to nuclear systems due to the existence of the nuclear force.
\end{abstract}

\section{Introduction}

Atomic nuclei are composed of protons and neutrons that interact with one another through the nuclear and electromagnetic forces. The former, which is much stronger than the latter, dominates the properties of atomic nuclei. Nevertheless, in specific studies, it is crucial to evaluate contribution of the latter to the properties of atomic nuclei. The mass difference of the mirror nuclei and energy of the isobaric analog state are such examples. In this report, we focus on the recent studies of the Coulomb energy density functionals (EDFs) of electron systems in the context of atomic nuclei $[1,2]$ in the density functional theory (DFT) $[3,4]$.

In the DFT for electron systems, the correlation energy is considered as well as the Hartree and exchange energies, whereas in the nuclear DFT it is not considered explicitly. The correlation EDF is tested for nuclear systems, where the local density approximation (LDA) is used, and we have used the experimentally observed charge-density distribution for quantitative calculations of selected nuclei to avoid an error coming from the density [1].

To calculate Coulomb exchange energy, we carry out self-consistent Skyrme Hartree-Fock calculations by using the Perdew-Burke-Ernzerhof generalized gradient approximation (PBE-GGA) Coulomb exchange functional [5] instead of using the exact-Fock term, and the optimal value of the free parameter $\mu$ that appears in the PBE-GGA functional is also discussed [2].

\section{Correlation Functional}

The Coulomb correlation energies calculated by the charge density distribution [6] in the LDA, $E_{\mathrm{Cc}}$, for selected nuclei from light to heavy region are shown in Table 1. For

*e-mail: naito@cms.phys.s.u-tokyo.ac.jp comparison, the Coulomb exchange energies calculated in the LDA, $E_{\mathrm{Cx}}$, and the ratio $E_{\mathrm{Cc}} / E_{\mathrm{Cx}}$ are also shown.

It is seen that in these calculations, $E_{\mathrm{Cc}}$ is all around $2 \%$ of $E_{\mathrm{Cx}}$. However, Bulgac and Shaginyan [7] evaluated that in atomic nuclei, $E_{\mathrm{Cc}} / E_{\mathrm{Cx}}$ would be around $-40 \%$ to $-20 \%$, instead of $2 \%$. Hypothetically, if there is only Coulomb interaction since correlation always further decreases the energy of the whole system, we have the signs of the Hartree, exchange, and correlation energies as $E_{\mathrm{Cd}}>0, E_{\mathrm{Cx}}<0$, and $E_{\mathrm{Cc}}<0$, respectively. In reality, the correlation EDF is not separable at all. In Ref. [7] the correlation EDFs are written in terms of the response functions, and such response functions are determined by the total interaction, i.e., mainly by the attractive nuclear part, instead of the repulsive Coulomb part. The total correlation energy is still negative, mainly due to the contribution of the nuclear interaction. As a result, the contribution of Coulomb interaction becomes positive, i.e., for the Coulomb energies, $E_{\mathrm{Cc}}$ has the different sign as $E_{\mathrm{Cx}}$. In short, the correlation energy density functionals of electron systems cannot be applied directly to atomic nuclei. It is also noted that the Coulomb correlation functional in the GGA gives around $30-80 \%$ of $E_{\mathrm{Cc}}$ in the LDA.

Table 1. Coulomb correlation energies $E$ cc for selected nuclei. Energies are shown in MeV. Data are taken from Ref. [1].

\begin{tabular}{rclc}
\hline \hline Nuclei & LDA $E_{\mathrm{Cx}}$ & LDA $E_{\mathrm{Cc}}$ & $E_{\mathrm{Cc}}^{\text {LDA }} / E_{\mathrm{Cx}}^{\text {LDA }}$ \\
\hline${ }^{16} \mathrm{O}$ & -2.638 & -0.05218 & $1.978 \%$ \\
${ }^{40} \mathrm{Ca}$ & -7.087 & -0.1329 & $1.875 \%$ \\
${ }^{48} \mathrm{Ca}$ & -7.113 & -0.1332 & $1.873 \%$ \\
${ }^{58} \mathrm{Ni}$ & -10.28 & -0.1879 & $1.828 \%$ \\
${ }^{116} \mathrm{Sn}$ & -18.41 & -0.3361 & $1.826 \%$ \\
${ }^{124} \mathrm{Sn}$ & -18.24 & -0.3356 & $1.840 \%$ \\
${ }^{208} \mathrm{~Pb}$ & -30.31 & -0.5524 & $1.823 \%$ \\
\hline \hline
\end{tabular}




\section{Exchange Functional}

The GGA Coulomb exchange functionals have been proposed as

$$
E_{\mathrm{Cx}}\left[\rho_{\mathrm{ch}}\right]=-\frac{3}{4} \frac{e^{2}}{4 \pi \varepsilon_{0}}\left(\frac{3}{\pi}\right)^{1 / 3} \int\left[\rho_{\mathrm{ch}}(\boldsymbol{r})\right]^{4 / 3} F(s(\boldsymbol{r})) d \boldsymbol{r},
$$

where $\rho_{\text {ch }}$ is the charge density distribution, $F$ is the enhancement factor depending on the density gradient, and $s$ denotes the dimensionless density gradient

$$
s=\frac{\left|\nabla \rho_{\mathrm{ch}}\right|}{2 k_{\mathrm{F}} \rho_{\mathrm{ch}}}, \quad k_{\mathrm{F}}=\left(3 \pi^{2} \rho_{\mathrm{ch}}\right)^{1 / 3} .
$$

In particular,

$$
F(s)=1+\kappa-\frac{\kappa}{1+\mu s^{2} / \kappa},
$$

is used in the PBE-GGA Coulomb exchange functional to satisfy some physical conditions, and $F \equiv 1$ corresponds to the LDA one, i.e., the Hartree-Fock-Slater approximation $[8,9]$. The parameter $\kappa=0.804$ is determined for any value of $\mu$ by the Hölder inequality [10]. In contrast, two different values of $\mu$ have been widely used in the studies of atoms [5] and solids [11], respectively. For the PBEGGA functional, $\mu=0.21951$ is determined by the random phase approximation of the homogeneous electron gas. Since this $\mu$ can be a different value for nuclear systems, the free parameter of the PBE-GGA Coulomb exchange functional, $\mu$, is multiplied by a factor $\lambda$. For the nuclear part, the SAMi functional [12] is used in the selfconsistent calculation. For comparison, the exact-Fock energies are also calculated [13].

The deviation of the Coulomb exchange energy $E_{\mathrm{Cx}}$ of the PBE-GGA or the exact-Fock from that of the LDA, $\Delta E_{\mathrm{Cx}}^{\mathrm{LDA}}$,

$$
\Delta E_{\mathrm{Cx}}^{\mathrm{LDA}}=\frac{E_{\mathrm{Cx}}-E_{\mathrm{Cx}}^{\mathrm{LDA}}}{E_{\mathrm{Cx}}}
$$

are shown as a function of mass number $A$ in Fig. 1.

It is found that in the light-mass region, to reproduce the exact-Fock results, $\lambda=1.50$ or more is required, while in the medium-heavy- and heavy-mass regions $\lambda=1.25$ reproduces well the exact-Fock results. The PBE-GGA result with $\lambda=1.00$ reproduces the exact-Fock result in the case of the super-heavy nucleus ${ }^{310} 126$ since the ratio of the surface region to the volume region in the superheavy nuclei is smaller than that in the medium-heavy or heavy nuclei.

\section{Conclusion}

In these works [1, 2], the Coulomb exchange and correlation EDFs in electron systems are applied to the nuclear systems. On the one hand, the Coulomb correlation energy density functionals of electron systems are not applicable for atomic nuclei, because these functionals are not separable and the nuclear interaction determines properties of atomic nuclei mainly. On the other hand, the PBEGGA Coulomb exchange functional with $\lambda=1.25$ reproduces the exact-Fock energy in the self-consistent Skyrme

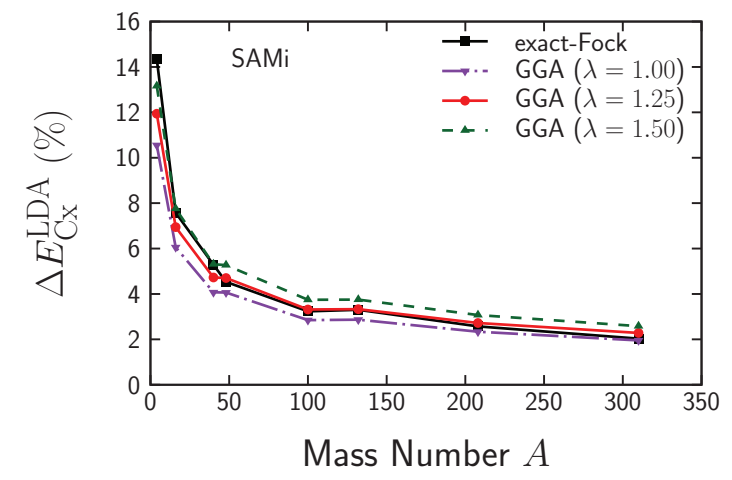

Figure 1. Deviation between the PBE-GGA and the LDA, $\Delta E_{\mathrm{Cx}}^{\mathrm{LDA}}$ defined as Eq. (3.4). For comparison the deviation between the exact-Fock and the LDA is also shown. Taken from Ref. [2].

Hartree-Fock calculations for atomic nuclei. It should be emphasized that the numerical cost of the self-consistent calculations with the PBE-GGA exchange functional is $O\left(N^{3}\right)$, whereas that with the exact-Fock term is $O\left(N^{4}\right)$. TN and HL would like to thank the RIKEN iTHEMS program and the JSPS-NSFC Bilateral Program for Joint Research Project on Nuclear mass and life for unravelling mysteries of the $r$-process. TN acknowledges the financial support from Computational Science Alliance, The University of Tokyo, Università degli Studi di Milano, and the JSPS Grant-in-Aid for JSPS Fellows under Grant No. 19J20543. HL acknowledges the JSPS Grant-in-Aid for Early-Career Scientists under Grant No. 18K13549. GC and XRM acknowledge funding from the European Union's Horizon 2020 research and innovation program under Grant No. 654002.

\section{References}

[1] T. Naito, R. Akashi, H. Liang, Phys. Rev. C 97, 044319 (2018)

[2] T. Naito, X. Roca-Maza, G. Colò, H. Liang, Phys. Rev. C 99, 024309 (2019)

[3] P. Hohenberg, W. Kohn, Phys. Rev. 136, B864 (1964)

[4] W. Kohn, L.J. Sham, Phys. Rev. 140, A1133 (1965)

[5] J.P. Perdew, K. Burke, M. Ernzerhof, Phys. Rev. Lett. 77, 3865 (1996)

[6] H. De Vries, C. De Jager, C. De Vries, At. Data Nucl. Data Tables 36, 495 (1987)

[7] A. Bulgac, V.R. Shaginyan, Nucl. Phys. A 601, 103 (1996); Phys. Lett. B 469, 1 (1999)

[8] P.A.M. Dirac, Proc. Camb. Phil. Soc. 26, 376 (1930)

[9] J.C. Slater, Phys. Rev. 81, 385 (1951)

[10] E.H. Lieb, M. Loss, Analysis, 2nd edn. (American Mathematical Society, 2001)

[11] J.P. Perdew, A. Ruzsinszky, G.I. Csonka, O.A. Vydrov, G.E. Scuseria, L.A. Constantin, X. Zhou, K. Burke, Phys. Rev. Lett. 100, 136406 (2008)

[12] X. Roca-Maza, G. Colò, H. Sagawa, Phys. Rev. C 86, 031306 (2012)

[13] X. Roca-Maza, L.G. Cao, G. Colò, H. Sagawa, Phys. Rev. C 94, 044313 (2016) 